\title{
ON CLOSED ADDITIVE SEMIGROUPS IN $E^{n}$
}

\author{
CHRISTOPH BANDELOW ${ }^{1}$
}

\begin{abstract}
Let $C(A)$ be the closed additive semigroup generated by a set $A \subset E^{n}$. A simple necessary and sufficient condition on $A$ for $C(A)$ to be a group is derived. An example which arose in the theory of random walks and stimulated these purely geometrical considerations is discussed at the end.
\end{abstract}

For an arbitrary subset $A$ of the $n$-dimensional Euclidean space $E^{n}$ $(n \geqq 1)$ let $S(A)$ denote the smallest additive semigroup containing $A, A^{-}$ the closure of $A$ with respect to the Euclidean topology, and $C(A):=$ $(S(A))^{-}$. Obviously, $C(A)$ is the smallest closed additive semigroup containing $A$, i.e., the closed additive semigroup generated by $A$.

Definition. $A \subset E^{n}$ is called omnilateral, if $A \neq \varnothing$ and for every hyperplane $H$ through the origin with $A \notin H$ there are points of $A$ in each of the two open half-spaces produced by $H$.

In other words, $A$ is omnilateral if $A \neq \varnothing$ and $u \in E^{n}, x \in A$ and $u x>0$ imply the existence of an $x^{*} \in A$ with $u x^{*}<0$. Some immediate consequences are listed in the following lemma.

Lemma. (1) $A$ is omnilateral if and only if $S(A)$ is omnilateral. (2) $A$ is omnilateral if and only if $A^{-}$is omnilateral. (3) An additive group $A$ is always omnilateral. (4) The image of an omnilateral set $A \subset E^{n}$ under a linear transformation from $E^{n}$ to $E^{m}$ is an omnilateral set in $E^{m}$.

We say a set $A \subset E^{n}$ is genuinely $n$-dimensional if there is no hyperplane through the origin 0 containing $A$. $A^{c}$ denotes the convex hull of $A$.

THEOREM 1. A genuinely $n$-dimensional set $A$ is omnilateral if and only if 0 is an inner point of $A^{c}$.

Proof. The if-part is trivial. Suppose, on the other hand, 0 is an exterior or a boundary point of $A^{c}$. There would be a hyperplane through

Received by the editors August 31, 1971.

AMS 1969 subject classifications. Primary 20M99, 50B99; Secondary 10E05, 52A20, $60 \mathrm{~J} 15$.

Key words and phrases. Closed additive semigroup in $E^{n}$, closed additive group in $E^{n}$, convex set, random walk.

1 This research was supported by NSF grant GU 2612 while the author was visiting the Department of Statistics of the Florida State University.

(c) American Mathematical Society 1972 
the origin such that one of the corresponding open half-spaces contains no point of $A[3, \mathrm{p} .20]$. Thus $A$ is not both omnilateral and genuinely $n$-dimensional.

THEOREM 2. $C(A)$ is a group if and only if $A$ is omnilateral.

Proof. The only-if-part follows from the Lemma, parts (1), (2) and (3).

To prove the if-part, one can assume that $A$ is genuinely $n$-dimensional. Let $0 \neq x_{0} \in C(A)$ and $\varepsilon>0$ be given. Since 0 is an inner point of $A^{c}$ there exists a $\lambda_{0}>0$ with $-\lambda_{0} x_{0} \in A^{c}$. Hence there exist a natural number $k$ and $k$ points $x_{1}, \cdots, x_{k} \in A$ and reals $\lambda_{1}>0, \cdots, \lambda_{k}>0$ with

$$
-\lambda_{0} x_{0}=\lambda_{1} x_{1}+\cdots+\lambda_{k} x_{k}
$$

By a theorem on approximations of real numbers by rational numbers (cf. for example $\left[4\right.$, p. 170]) there exist rational numbers $p_{i} / q(i=0, \cdots, k$; $p_{i}$ and $q$ natural numbers) with

$$
\left|p_{i}\right| q-\lambda_{i} \mid<\left(q \cdot q^{1 /(k+1)}\right)^{-1} \quad(i=0, \cdots, k),
$$

where the common denominator $q$ can be chosen arbitrarily large. If

it follows that

$$
\frac{1}{q^{1 /(k+1)}}<\frac{\varepsilon}{(k+1) \max \left(\left|x_{0}\right|, \cdots,\left|x_{k}\right|\right)},
$$

and thus

$$
\left|\left(p_{i}-\lambda_{i} q\right) x_{i}\right|<\varepsilon /(k+1) \quad(i=0, \cdots, k)
$$

$$
\left|\sum_{i=0}^{k} p_{i} x_{i}\right|=\left|\sum_{i=0}^{k}\left(p_{i}-\lambda_{i} q\right) x_{i}\right|<\varepsilon .
$$

But $y(\varepsilon):=\left(p_{0}-1\right) x_{0}+p_{1} x_{1}+\cdots+p_{k} x_{k} \in C(A)$ and thus $\lim _{\varepsilon \downarrow 0} y(\varepsilon)=$ $-x_{0} \in C(A)$. Q.E.D.

EXAMPLE. Let $X_{1}, X_{2}, \cdots$ be a sequence of independent, identically distributed $n$-dimensional random vectors and $S_{r}:=X_{1}+\cdots+X_{r}$ $(r=1,2, \cdots)$ the associated random walk. An $x \in E^{n}$ is called possible if $P\left(\left|S_{r}-x\right|<\varepsilon\right.$ for some $\left.r\right)>0$ for each $\varepsilon>0$ ([2], [1]). The set $C$ of all possible $x$ is obviously the closure of the additive semigroup generated by the set

$$
A:=\left\{x \in E^{n}: P\left(\left|X_{1}-x\right|<\varepsilon\right)>0 \text { for each } \varepsilon>0\right\} .
$$

$A$ is omnilateral, if $X_{1}$ has expectation 0 . Indeed, if $A$ is not omnilateral there exists a $u \in E^{n}$ with $u x \geqq 0$ for all $x \in A$ and $u y>0$ for some $y \in A$. Assuming that the expectation $e$ of $X_{1}$ exists and denoting the distribution 
function of $X_{1}$ by $F_{X_{1}}$, we get

$$
u e=u \int_{E^{n}}\left(x_{1}, \cdots, x_{n}\right) d F_{X_{1}}\left(x_{1}, \cdots, x_{n}\right)=\int_{A} u x d F_{X_{1}}\left(x_{1}, \cdots, x_{n}\right)>0
$$

i.e. $e \neq 0$.

Thus, by Theorem 2, the set of all possible $x$ of a random walk with expectation 0 is a group.

\section{REFERENCES}

1. C. Bandelow, Recurrence properties of functionals of Markov chains, Z. Wahrscheinlichkeitstheorie und Verw. Gebiete 19 (1971), 1-18.

2. K. L. Chung and W. H. J. Fuchs, On the distribution of values of sums of random variables, Mem. Amer. Math. Soc. No. 6 (1951), 1-12. MR 12, 722.

3. H. G. Eggleston, Convexity, Cambridge Tracts in Math. and Math. Phys., no. 47, Cambridge Univ. Press, New York, 1958. MR 23 \#A2123.

4. G. H. Hardy and E. M. Wright, An introduction to the theory of numbers, 3rd ed., Clarendon Press, Oxford, 1954. MR 16, 673.

Department of Statistics, Florida State University, Tallahassee, Florida 32306

Current address: Institut für Mathematik, Ruhr Universität, 463 Bochum, Federal Republic of Germany 